\title{
El magisterio mexicano y el SNTE ante las transformaciones del trabajo docente en México
}

\author{
Mexican teachers and SNTE before the transformation of \\ teching in Mexico
}

José David Alarid Dieguez ${ }^{1}$

\section{Resumen}

En México durante los últimos años se han puesto en marcha una serie de reformas en el sector de la educación básica, desde el Estado se ha argumentado que aquellas tienen por objetivo mejorar la calidad de la educación. La más reciente llamada "Alianza por la Calidad de la Educación" fue firmada por el ejecutivo nacional y la dirigencia del Sindicato Nacional de los Trabajadores de la Educación (SNTE) en 2008. Ante esta situación varios miles de maestros expresaron su descontento que se materializó en movilizaciones. Los profesores inconformes argumentan que la 'alianza' representa la puesta en acción de transformaciones que deterioran sus condiciones de trabajo. Aducen que algunas de las medidas contempladas en ella significan empobrecer la práctica educativa y argumentan que el proceso se ha dado al margen de su opinión, de hecho, en contra de ella, lo que supone que el SNTE no representa sus intereses. En el presente trabajo se analizan algunas consecuencias de la reforma educativa denominada Alianza por la Calidad de la Educación para el trabajo docente y la forma en que el SNTE ha participado en el proceso contra la opinión de una parte importante de los maestros a los que formalmente representa. Palabras-clave: SNTE; Alianza por la Calidad de la Educación; Reestructuración laboral.

\begin{abstract}
In Mexico in recent years have launched a series of reforms in the basic education sector, since the State has argued that these are intended to improve the quality of education. The latest so-called "Alliance for Quality Education" was signed by the national executive and the leadership of the National Union of Education Workers (SNTE) in 2008. In this situation several thousand teachers expressed dissatisfaction that materialized in public demonstrations. The protesters argue that teachers 'alliance' is put in action the changes that impair their working conditions. They argue that some of the measures contained in it means educational impoverishment and argue that the process is given regardless of their opinion, in fact, against it, which means that the SNTE not represent their interests. In this paper we analyze some consequences of educational reform known as the Alliance for Quality Education for teaching work and how the SNTE has participated in the process against the advice of an important part of teachers who formally represents.
\end{abstract}

Key-words: SNTE; Alliance for Quality Education; Labor restructuring. 


\section{Introducción}

En mayo de 2008 se firmó por parte del Gobierno Federal (GF) y el Sindicato Nacional de Trabajadores de la Educación (SNTE) la alianza por la calidad de la Educación ACE. Entre gran despliegue promocional se presentó a la ACE como un parteaguas para "elevar la calidad de la educación en México", sin embargo desde distintos lugares diversos actores interesados en el ámbito educativo realizaron una serie de señalamientos y críticas a la ACE. A continuación presentamos el contexto en el cual se presentó la ACE y los antecedentes de ésta, en particular analizaremos el Acuerdo Nacional para la Modernización de Educación Básica y Normal (ANMEB), de la cual la ACE es un actualización y profundización. Posteriormente pasaremos a analizar Algunos aspectos de la propia Alianza y sus consecuencias para el trabajo docente. Por último realizaremos algunas consideraciones acerca del papel del SNTE en el proceso.

\section{Las reformas de la Educación Básica: el ANMEB y la ACE}

La política educativa vigente en México desde cuando menos 1992 se ha dirigido a entre otras cosas a 'eficientar' el gasto educativo, 'hacer más con menos' ha sido uno de los lemas favoritos que han guiado las reformas implementadas en México desde la década de los 80. En un contexto en el que el discurso empresarial ha ido un ganando lugar prominente en los discursos educativos oficiales.

En 1992 se firmó el Acuerdo Nacional para la Modernización de la Educación Básica (ANMEB) (MÉXICO, 1992) en donde se concretó el plan modernizador transexenal para el sector de la educación básica del país.

El ANMEB fue firmado por el Gobierno Federal, los gobiernos estatales de la república y el Sindicato Nacional de Trabajadores de la Educación con el compromiso de: extender la cobertura de los servicios educativos, elevar la calidad de la educación, uso de recursos presupuestales crecientes para la educación pública, reorganización del sistema educativo, reformulación de los contenidos y materiales educativos y la revaloración de la función magisterial. Temas, por cierto, reiterados en la ACE.

En el Acuerdo quedó plasmado explícitamente que los gobiernos estatales reconocen al SNTE como el titular de las relaciones laborales colectivas de los trabajadores de base que se incorporan al sistema educativo estatal. También, se hizo hincapié en favorecer 'la nueva participación social', para lo cual considera importante fortalecer la capacidad de organización y la participación desde las escuelas de padres de familia. Se perfilaba, desde el punto de vista de sectores de maestros, la posible implementación de un dispositivo control de padres de familia sobre la labor de los docentes, de forma tal que se garantizara el cumplimiento de planes y programas educativos. Temas también reiterados en la ACE.

\section{La Carrera Magisterial}

En el documento se anunció la creación de la Ilamada Carrera Magisterial (CM) Tal vez, la parte más importante del Acuerdo, junto con la descentralización, en términos de sus consecuencias para el sector.

Se señala que la CM daría respuesta a dos necesidades de la actividad docente. En términos de las transformaciones de trabajo de los maestros, la carrera magisterial representa un hito importante por las consecuencias de la implementación de este programa en las actividades cotidianas de los maestros de educación básica y en su relación con su sindicato.

La descentralización fue una línea central de las reformas educativas implementadas y a partir del ANMEB se transfirieron los servicios educativos a los estados de la república, con la excepción del D. F., asiento de la capital política del país. Gobernada desde 1997 por el centro izquierda.

La CM aparece como un plan de reestructuración de las condiciones de trabajo y promoción de los profesores, que transformaría las viejas formas de gestión de la fuerza de trabajo magisterial. 
Podría resumirse al ANMEB como una política desde el Estado que planteó la financiación la educación vía la federalización -descentralización y la reorganización del trabajo magisterial para adecuarlo a los parámetros de la 'flexibilidad' laboral.

El trabajo cotidiano de los docentes de educación básica está regulado por directrices emitidas centralmente desde la Secretaría de Educación Pública (SEP) que se concretan en el currículo oficial de la educación básica y otros ordenamientos. En esa perspectiva es que situamos al establecimiento de políticas evaluación como uno de los elementos centrales de la implementación de los procesos de reestructuración del trabajo docente derivados de la modernización educativa, en gran medida inspiradas por la OCDE.

El establecimiento de la Carrera Magisterial (CM) ha significado un impulso en la importancia de la evaluación para el control del trabajo magisterial. Así, se estableció en los lineamientos de CM, que para lograr ingreso y/o promociones en ella, se tomaría en cuenta el resultado promedio de exámenes aplicados muestras de alumnos de los profesores para evaluar su aprovechamiento. Con ese indicador se intenta medir la 'eficiencia' en el trabajo docente. Un propósito de esa condición es intentar estandarizar la labor de los maestros no sólo en tiempos sino también en contenidos, dado que para poder obtener los puntos asignados en ese rubro el maestro necesita programar sus actividades de tal forma que se sincronicen con las evaluaciones programadas, lo que implica que su programación se sujeta a tiempos externos más que atender al ritmo de aprendizaje de sus alumnos, lo cual desde el punto de vista psicopedagógico, es lo óptimo para poder favorecer el desarrollo de aprendizaje de tipo 'significativo'. (COLL, 1990). Esta situación le supone al docente aumento de estrés y sobrecarga de trabajo. Sus criterios se vuelven más cuantitativos que cualitativos en la evaluación de su trabajo y del desempeño de sus alumnos.

Por otro lado, los resultados de las evaluaciones se utilizan como indicadores de desempeño para diferenciar a los 'buenos' de los 'malos' maestros, sin que necesariamente esos resultados reflejen, desde la perspectiva de los profesores, la calidad de la labor desarrollada (ALARID, 2001). La carrera magisterial tiene como resultado la diferenciación de las remuneraciones que perciben los maestros. A la fecha existen cinco niveles de ingresos: sin ingresar a CM, niveles a, b, c y d. Las diferencias de ingresos salariales entre cada uno de estos niveles es de, aproximadamente, 33\%; por lo cual un profesor que se encuentre en el nivel "c" percibirá el doble que lo recibe un profesor que no ha ingresado al programa. Sin duda que esto marca notables diferencias de ingresos entre quienes componen la planta docente de las escuelas.

Es de hacer notar que, se comenta entre los profesores que, en distintos momentos de los procesos de evaluación existe corrupción. Por ejemplo, es conocido entren los profesores que los exámenes se venden en el "mercado negro" y que hay quienes los consiguen y obtienen ventajas sobre quienes los presentan sin recurrir a esa artimaña. Esta situación supone para ellos un elemento de negación de la objetividad pretendida en la evaluación y, por tanto, su descalificación ${ }^{2}$ Lo que aunado a inconformidades respecto de las formas en que los órganos de evaluación, presididos por los directores de las escuelas suelen evaluar y, por tanto asignar puntos, que incide en la calificación total y en las posibilidades de ingresar y /o promoverse en la CM. podemos afirmar, con base en entrevistas realizadas, que para un sector importante de los docentes la CM no refleja la calidad de la labor efectuada en el día a día y lo interesante es que esto es opinado aún por profesores que están en niveles altos, "C" o "D", de CM y que, por tanto, pueden ser considerados como los 'ganadores' de la situación. En resumen, existe entre importantes sectores de los profesores de base gran inconformidad con distintos aspectos de la CM.

Cabe hacer notar que el programa de Carrera Magisterial (CM) tiene límites presupuestales por

2 Situación que fue corroborada el año pasado cuando el diario de circulación nacional, La Jornada, publicó uno de los exámenes que se iban a aplicar y que había sido comprado en el mercado negro, lo que motivó que se suspendiera y pospusiera la aplicación del examen ya programado. 
lo cual el acceso a la misma está dado por los techos presupuestales asignados. Esto significa que el criterio de acceso es de cupo y no de puntajes, es decir ingresan tantos docentes como la bolsa asignada lo permita y no todos aquellos que cubran un parámetro determinado.

La CM impone nuevas condiciones de trabajo al fomentar la intensificación, apunta simultáneamente a aumentar el control central de la materia de trabajo del docente, disciplinándolo a través de incentivos económicos y a minar la identidad gremial y profesional de los maestros.

Con lo hasta aquí planteado al respecto de $\mathrm{CM}$ es posible adelantar que las consecuencias de ese programa rebasan, por mucho, las afirmaciones de que, tal programa, es una forma de lograr 'mejorar' la calidad de la labor docente, al mismo tiempo que de mejorar las remuneraciones económicas de los maestros.

\section{La Alianza para la Calidad de La Educación (ACE)}

En este apartado analizaremos algunos de los aspectos de la ACE que afectan directamente al trabajo docente. El análisis de otros aspectos de la ACE quedan fuera del propósito del presenta trabajo.

Firmada por el Gobierno Federal (GF) y el SNTE en mayo de 2008, la ACE se presenta con el objetivo transformar la calidad de la educación que ofrece el Sistema Educativo Nacional, para ello el Gobierno Federal y el SNTE ‘agendaron' una serie de compromisos. Afirmaron que "realizaron 'amplias' consultas y formulron propuestas plasmadas en: el Plan Nacional de Desarrollo, el Programa Sectorial de Educación y el Cuarto Congreso Nacional y Segundo Encuentro Nacional de Padres de Familia y Maestros del Sindicato Nacional de Trabajadores de la Educación, lo que sería la base de la ACE. Cabe hacer notar, que profesores entrevistados afirman que no fueron consultados y que no conocían a profesores que hubiesen sido consultados.

La propuesta contempla una serie de rubros: Infraestructura y equipamiento, de las Tecnologías de la información y la comunicación, Gestión y participación social, salud, alimentación y nutrición, desarrollo social y comunitario, centros escolares, reforma curricular, maestros, alumnos y evaluación.

Entre los acuerdos se encuentra los relativo a la 'profesionalización' de los maestros y autoridades educativas para lo cual se propone la creación de un "Sistema Nacional de Formación Continua y Superación Profesional de Maestros en Servicio, participación de instituciones de educación superior brindando cursos a maestros; certificación independiente de 'competencias profesionales'; de manera destacada se plantea que 'Los profesores cuyos estudiantes muestren bajo rendimiento en pruebas estandarizadas como la prueba Evaluación Nacional del logro Académico en los Centros Escolares (ENLACE) deberán tomar cursos especialmente orientados', también se propone mejorar la 'calidad' del personal de las escuelas normales, las escuelas de formación inicial de los profesores de educación básica, y crear cinco centros regionales de 'excelencia académica'.

Se planteó reformar los lineamientos de CM para que se considerasen de manera exclusiva tres factores: 'aprovechamiento escolar (medido a través de instrumentos estandarizados aprobados por el Sistema Nacional de Evaluación de la Educación), cursos de actualización (certificados independientemente) y desempeño profesional'. Por lo cual factores como antigüedad y preparación profesional quedan fuera.

Se trataría de 'fortalecer la calidad' y 'estimular el mérito individual de los maestros en función exclusiva de los resultados de logro de sus alumnos'. Lo que supone esos resultados, las calificaciones de los alumnos, tendrían como factor determinante el trabajo magisterial, con lo que se deja de lado el análisis de las condiciones en las que tal trabajo ocurre.

En la ACE replantea que la escuela debe ser promotora una educación basada en valores, de calidad que impulse la 'construcción de ciudadanía y, que dé 'impulso a la productividad y la promoción de la competitividad'.

Se pretende 'impulsar' la reforma de enfoques, contenidos y asignaturas de la educación básica. Se plantea que el inglés se debe enseñar desde preescolar.

La evaluación aparece como uno de los pilares de la ACE, con miras a elevar la calidad 
de la educación, facilitar la rendición de cuentas y servir de base al diseño de políticas educativas. Se pretende articular el 'Sistema Nacional de Evaluación'; realizar evaluación 'exhaustiva' de todos los actores del proceso educativo; establecer estándares de desempeño para los distintos componentes del Sistema Educativo Nacional basados en 'parámetros y criterios de desempeño internacionales'. Todo esto a partir del ciclo escolar 2008- 2009. (SEP, 2008)

\section{Consecuencias de la ACE para el trabajo docente}

Son múltiples los rubros en los que la ACE afecta al trabajo magisterial, a continuación presentamos algunos de ellos.

La contratación y la ACE. Para poder aspirar a una plaza se necesita aprobar un examen de oposición, con lo que elementos como la antigüedad quedan fuera. Esto supone que maestros que tienen años impartiendo clases pueden quedar fuera por obtener puntajes 'bajos' en el examen. A primera vista la situación puede parecer pertinente, sin embargo queda por establecer la idoneidad del instrumento para delimitar las posibilidades de ser un maestro exitoso. Es decir, difícilmente, una prueba de papel y lápiz logrará evidenciar las aptitudes ante un grupo que un solicitante pueda tener. Asimismo, señalan algunos profesores entrevistados que existe un número de plazas que le son otorgadas al Comité Ejecutivo Nacional del SNTE para que éste las distribuya de manera discrecional, como tradicionalmente lo ha hecho.

Entre los cambios en la CM que se impulsaron a parir de la ACE están los factores de evaluación, desaparecen antigüedad y preparación profesional. En lo tocante al primero de ellos se elimina un elemento que durante años representó una marca de identidad magisterial, los años en el puesto que era visto como indicador de experiencia.

En lo que respecta al segundo elemento, la preparación profesional, vale la pena hacer algunas consideraciones. Durante algún tiempo se impulsó la 'profesionalización' de la profesión docente, como parte de esa situación se alentó que los docentes realizaran estudios de posgrado, los que se traducían en algunos puntos para la CM. Profesores entrevistados para este trabajo afirman que ese el puntaje otorgado alentó a algunos maestros a cursar tal tipo de estudios. Sin duda, cuando menos en algunos casos, esos estudios se tradujeron en mejores desempeños en la docencia. Sin embargo, la nueva disposición los desalentará. Como afirma una profesora, "¿de qué sirve prepararse? Yo tengo maestría y no me la toman en cuenta. Muchos profesores se prepararon por CM."

En su lugar se empiezan a ofertar curso que ofrecen distintas instituciones privadas y públicas. Por ejemplo, en el estado de Morelos, se ofertaron cursos para la materia de Español que ofreció el ITESM y que se ofrecían gratuitamente a los profesores de la materia aludida. El requisito era que cubrieran todas las horas programadas, pues en caso contrario se les cobraría el curso, por lo cual muchos profesores decidieron rechazarlo, dado que corrían el riesgo de que por diversas circunstancias, terminasen pagando algo que ni siquiera era algo que ni habían elegido.

Los cursos aludidos señalan un aspecto que maestros y especialistas han señalado como uno de los objetivos no declarados de la ACE: la privatización, de diversas esferas de la educación pública. En este caso de la actualización, concediendo contratos a universidades privadas.

El desempeño de los alumnos se convierte en el indicador del trabajo magisterial. Esta situación alienta que la atención a la diversidad pueda convertirse en un problema para algunos maestros. Tal es el caso de aquellos que tienen en sus grupos a niños 'especiales', con 'necesidades educativas especiales'. Pues como dice una entrevistada, "Esos niños bajan el promedio, y así no vas a pode r acceder a los puntajes para promoverte."

De igual manera, los profesores adscritos a zonas menos favorecidas o incluso a turnos vespertinos, a los cuales tradicionalmente se asignan a los niños con menores rendimientos o con rezago escolar, se sienten en desventaja y muchos intentarán eludir tales escuelas.

Otro de los puntos controvertidos de la ACE lo constituye la instauración de un estímulo económico para aquellos maestros cuyos alumnos obtengan rendimientos 'más altos' en la 
prueba Evaluación Nacional del logro Académico en los Centros Escolares (ENLACE).

Esta medida tiene una serie de implicaciones que vale la pena resaltar, parte del supuesto implícito que el trabajo magisterial constituye el elemento central en el rendimiento de los alumnos. Lo que sobresimplifica la relación maestro- alumno- conocimiento; sin duda que los rendimientos de los alumnos suelen estar correlacionados con la dinámica enseñanzaaprendizaje en la que el profesor tiene un papel preponderante. Sin embargo, la proporción de la varianza de la que ese factor es responsable no puede hacerse pasar cómo el único o incluso como el principal elemento responsable del nivel de logro de aprendizaje de los alumnos. Con el problema adicional que supone definir los tipos de aprendizaje que pueden medir los exámenes de opción múltiple que se aplican a los alumnos en pruebas como ENLACE. Partiendo del supuesto que una serie de aprendizajes de tipo procedimental y actitudinal no son susceptibles de ser evaluados adecuadamente a través de esos medios. De tal manera que, en el mejor de los casos se podrá determinar a través del tipo de evaluación sumaria que suponen las pruebas estandarizadas 'objetivas' sólo una parte, no necesariamente la más relevante, de los aprendizajes escolares.

En la medida en que los resultados de ENLACE se convierten en un elemento central de la definición de la 'calidad' de la educación brindada por los profesores, el horizonte del trabajo magisterial se ve redireccionado hacia el logro más alto posible en los resultados de la evaluación. En una perspectiva indeseable de 'enseñar para el examen' (Whitty, 1999), dejando de lado una perspectiva de la educación más rica y más amplia.

La perspectiva que se ha impuesto sitúa a la evaluación como instrumento de obtención de datos que permitan el control del personal docente para poder administrar su trabajo de una manera más "eficiente" en la perspectiva de de estratificarlos y diferenciar el pago que se les hace, legitimando, al mismo tiempo, la exclusión de muchos de ellos de ingresos adecuados. En relación con los resultados obtenidos por los alumnos en el ciclo escolar próximo pasado. Sin duda esta situación abre posibilidades a un cúmulo de efectos no deseados, pero previsibles como el que los docentes busquen obtener el estímulo a través del entrenamiento de los alumnos en la resolución de exámenes estandarizados, dejando de lado contenidos fundamentales para el desarrollo de los alumnos, pero no susceptibles de ser cuantificables desde los parámetros de las pruebas estandarizadas.

\section{Los Maestros y el SNTE}

La reestructuración de las condiciones del trabajo magisterial que supone la ACE es demasiado importante como para ser indiferentes a la masa de profesores de base. Si el sindicato fuese una instancia de representación de intereses de los maestros de base sería menester que hubiese instancias de participación de ellos en la toma de decisiones, de tal manera que se lograse un acuerdo que favoreciera la aquiescencia de los principales artífices, desde la óptica implícita en el diseño de las reformas, de la consecución del objetivo de 'elevar la calidad de la educación'. Sin embargo, nada más alejado de la realidad, la ACE, como todas las reformas educativas se han implantado no sólo sin la participación de los maestros, ni siquiera a través de consultas y/o campañas de convencimiento, sino en contra de su manifiesta oposición.

EI SNTE monopoliza la representación de los maestros del país y el Estado mexicano lo reconoce oficialmente como único interlocutor legal para tratar los asuntos del magisterio.

La dominación en el SNTE 'debe' entenderse en gran medida por el apoyo del Estado al CEN; sin embargo, esto no significa que sea la amenaza del uso de la fuerza y la imposición directa lo que impide la expulsión de la 'elite' que domina al SNTE. Muchos miembros del SNTE no se sienten impelidos a cambiar de manera urgente a la 'dirigencia'. Por otro lado, eso no significa que los 'maestros se sientan adecuadamente representados por el CEN.

Aquíse puede abrir el problema de la 'legitimidad' en el manejo y dominio político en el SNTE. Después de 1989 el sindicato ha reformado dos 
veces sus estatutos, los que han sido adecuados a las necesidades del dominio del grupo dominante. Sin embargo, es importante hacer notar que ni los estatutos readecuados a las necesidades de la corriente hegemónica son respetados.

En esa línea, hay que subrayar la ausencia de participación de las 'bases' en la conducción de los asuntos del sindicato tales como la elección de 'dirigentes' y cambios de estatutos.

Los maestros de base no son consultados en la toma de decisión, en procesos como las negociaciones salariales, procesos en los que los 'pliegos petitorios' nunca son conocidos, ni mucho menos puestos a consideración por parte de la base. Por supuesto, tampoco decidir si el SNTE los compromete con políticas educativas que, desde su punto de vista pudiesen resultar lesivas para sus interese o los de la educación pública.

El SNTE maneja una cantidad de dinero estimada en 2, 000 millones de pesos anuales de cuotas que recibe directamente, descontadas por nómina a los profesores, es decir no son pagados voluntariamente por sus agremiados. No existe un control de tal cantidad, ni alguna rendición de cuentas. Lo que confiere a la 'dirección' del sindicato una ingente cantidad de recursos que se han utilizado de manera discrecional y patrimonialista para incrementar su capital político.

Con la situación de deterioro salarial ha que se han visto sometidos los trabajadores de la educación, existe una erosión de las bases de legitimidad que el SNTE mantiene ante ciertos sectores de la 'base magisterial'.

Si bien entre el Ejecutivo Federal y la dirección del SNTE subsisten diferencias de criterios al respecto del modo de operar la modernización; tales diferencias son negociadas dentro de los entretelones del sistema (neo)corporativo,.

Por su lado, los maestros disidentes al grupo dominante en el SNTE agrupados en la Coordinadora Nacional de los Trabajadores de la Educación (CNTE) se manifiestan como contrarios a las políticas 'modernizadoras' de corte 'neoliberal' que afectan al sector, se han movilizado para resistir y contrapesar las medidas que se consideran contrarias a los 'intereses del magisterio' y la educación pública.
Para poder organizarse independientemente los maestros 'disidentes' han implementado formas organizativas propias, lo que les ha permitido implementar sus decisiones de manera directa, sin tener que pasar por la anuencia de la corriente dominante.

Cabe hacer resaltar que la dirigencia del SNTE fue una de las principales promotoras de las reformas a la ley del Instituto de Seguridad Social y Servicios de los Trabajadores del Estado (ISSSTE), a contracorriente de los deseos de la gran mayoría de los agremiados del SNTE.

La reacción de los trabajadores de base del SNTE y de otros sindicatos ha sido de resistencia ante una medida que sin duda vulnera derechos y que se traduce en mayores cargas financieras para los trabajadores y menores expectativas de remuneración al momento de la jubilación, así como perdida de prestaciones, en suma, perdidas a cambio de ganancias para las camarillas dirigentes, quienes sacrifican a sus agremiados y sus intereses desde el discurso de el sacrificio es en nombre de los intereses superiores del país.

El descontento generado por las reformas a la Nueva Ley del ISSSTE y la ACE propiciaron la movilización, aun en sectores que no habían mostrado descontento contra la dirigencia del SNTE, ante su papel no sólo como promotor sino como abierto ganador de privilegios económicos y políticos en contra de los intereses de sus afiliados a los que, por supuesto, no consultó.

Ante esta situación, la cuestión de la legitimidad de la dirección del SNTE se avizora como cada vez más frágil, pero la dirección del sindicato sigue siendo sostenida por el Estado mexicano. De manera clara en algunas entidades del país, siendo los casos más visibles las secciones sindicales de Michoacán y Oaxaca existe un abierto rechazo mayoritario a la dirigencia del sindicato.

Con motivo de la puesta en marcha de la ACE la sección 19 del estado de Morelos que la que se movilizó de manera masiva para pedir la derogación de la Alianza. Si bien otros contingentes se movilizaron en distintas entidades federativas tales como Quintana Roo, Puebla, Michoacán, Guerrero, D. F. y otros, ninguno con la decisión de Morelos. 
Ante las reformas y sus efectos los maestros de educación básica se han visto obstaculizados para enfrentar de manera organizada la situación, vivida por la mayoría de ellos como de deterioro de sus condiciones laborales y sus prestaciones.

La participación de los maestros ha sido bloqueada sistemáticamente por el Comité Ejecutivo Nacional (CEN) del SNTE. Podemos afirmar que la representación legal que ostenta la cúpula representa una expropiación de la posibilidad de auto representarse. Por supuesto, los profesores desconocen la manera en que la élite del CEN toma las decisiones.

Así, las negociaciones del CEN con la SEP transcurren en la más absoluta secrecía y discrecionalidad. Los resultados no son nunca puestos a consulta, por tanto, todo queda sujeto a las negociaciones y decisiones de una cúpula alejada de las preocupaciones de los profesores de base.

\section{A manera de conclusiones}

La ACE representa una actualización y profundización de las reformas educativas implementadas desde la década de los ochenta desde la óptica neoliberal. A pesar del poco éxito, si es que alguno, de los programas puestos en marcha desde el ANMEB y la ACE incluida, se sigue insistiendo por parte del Gobierno Federal y el SNTE en más de lo mismo.

Las consecuencias para el trabajo magisterial son relevantes y avanzan en la línea del deterioro de las condiciones laborales de los profesores en detrimento de su actuación, lo que resulta inquietante en términos del menoscabo de la relevancia de la educación que los niños mexicanos que concurren a escuelas públicas reciben.

Por su parte el grupo dominante en el SNTE mantiene una agenda propia, ajena a los intereses de la mayoría de sus agremiados, probablemente teniendo claro de que sus bases de sustento no se encuentran en su representatividad ante sus agremiados, sino se fincan en sus vínculos con los primeros círculos del poder estatal. Razón por la cual las inconformidades manifiestas por la base magisterial no sólo no las ha atendido sino que ha adoptado el papel de elemento de contención y en su momento de represor de las protestas magisteriales.

\section{Referências Bibliográficas}

ALARID, D. Reestructuración de la educación básica y proceso democrático en la sección del SNTE. en: De la Garza, E. (Coord.). Democracia y cambio sindical en México. México: UAM- Y - Plaza y Valdez, 2001.

APPLE, M. Maestros y textos. Barcelona: Paidós, 1989. Educación y poder. Barcelona: Paidós, 1995.

ARONOWITZ, S. The knowledge Factory. Boston: Beacon Press, 2000.

ARNAUT, A. Historia de una profesión. Los maestros de educación primaria en México, 1887-1994. México, CIDE, 1996.

BALL, S. La micropolítica de la escuela. Barcelona: Paidós, 1989.

Foucault y la educación: disciplinas y poder. Madrid: Morata, 1997.

BANCO MUNDIAL. El financiamiento de la educación en los países en desarrollo. Washington: Banco Mundial, 1995.

BONAL, X. Globalización y política educativa: un análisis crítico de la agenda del Banco Mundial para América Latina. Revista Mexicana de Sociologia, Instituto de Investigaciones Sociales de la UNAM, v.64, n. 3, jul./ sept. 2002.

BOURDIEU, P. Sociología y cultura. México: CNCA- Grijalbo, 1990.

COLL, C. et al. Desarrollo psicológico y educación Il: psicología de la educación. Madrid: Alianza, 1990.

COOK, L. Organizing dissent: unions, the state, and the democratic teachers' movement in Mexico. Pennsylvannia: PSU Press, 1996. 
DÍAZ BARRIGA, A. Universitarios: institucionalización académica y evaluación. México: CESU- UNAM, 1997. Carrera magisterial y evaluación de profesores: entre lo administrativo y lo pedagógico en M. Rueda y M. Landesmann, ¿Hacia una nueva cultura de la evaluación de los académicos? México: CESU/UNAM, 1999. DE LA GARZA, E. Ascenso y crisis del estado social autoritario. México: COLMEX, 1988.

(Coord.). Crisis y sujetos sociales en México. México: Porrua, 1992.

(Coord.). Políticas públicas alternativas en México. México: La Jornada- CIICH- UNAM, 1996. (Coord.). La formación socioeconómica neoliberal. México: Plaza y Valdés, 2000a.

GIDDENS, A. . La constitución de la sociedad. Buenos Aires: Amorrortu, 1995.

GIMÉNEZ, G. La teoría y el análisis de la cultura. Problemas teóricos y metodológicos. en: González; Galindo, J. (Coord.). Metodología y cultura. México: CNCA, 1994.

GIROUX, H. Educación y poder. Barcelona: Paidós, 1990.

GRAMSCI, A. Cuadernos de la cárcel. México: Juán Pablos, 1975. Tomo 1.

HARGREAVES, A. Profesorado, cultura y postmodernidad. Cambian los tiempos cambia el profesorado. Madrid, Morata, 1996

HARVEY, D. The condition of posmodernity. Balden, USA: Blackwell, 1990.

IBARROLA, M. et al. ¿Quiénes son nuestros maestros? México: Fundación SNTE, 1997.

JIMÉNEZ, L. La reestructuración de la escuela y las nuevas pautas de regulación del trabajo docente. Revista mexicana de investigación educativa, v. 8, n. 19, sept./dic. 2003.

MÉXICO SECRETARÍA DE EDUCACIÓN PÚBLICA (SEP). Lineamientos Generales de Carrera Magisterial. México, SEP, 1998.

MÉXICO. PODER EJECUTIVO FEDERAL. Acuerdo Nacional para la Modernización de la Educación Básica y Normal (ANMEB). México: PEF, 1992.

MÉXICO. PODER EJECUTIVO FEDERAL. Plan nacional de desarrollo: 1977- 1982. México: PEF, 1977.

MÉXICO. PODER EJECUTIVO FEDERAL. Plan para la modernización educativa: 1989- 1994. México: PEF, 1989. POPKEWITZ, T. S. Sociología política de las reformas educativas. Madrid: Morata, 1998.

SMYTH, J. Teachers Work in a Globalizing Economy. Londres: Falmer Press, 2000.

STREET, S. Maestros en movimiento. México: CIESAS, 1992.

STREET, S. "El movimiento magisterial como sujeto democrático ¿autonomización de los educadores o ciudadanización de la educación?". El cotidiano Vol. 14, No.87, México, Universidad Autónoma Metropolitana, enero- febrero de 1998.

STREET, S. Trabajo docente y poder de base en el sindicalismo magisterial en México. en: La Ciudadanía Negada. Buenos Aires: CLACSO, 2000.

STREET, S. Trabajo docente y reforma escolar en América Latina. Washington, USA: [s.n.], 2001. Documento presentado en encuentro de la Latin American Studies Asociation.

TEDESCO, J. C. Nuevas estrategias de cambio educativo en América Latina. en: TEDESCO, J. C. et al. Necesidades básicas de aprendizaje: Estrategias de acción. Santiago de Chile, UNESCO/IDRC, 1993.

THOMPSON, J. Ideología y cultura moderna. México: UAM- Xochimilco, 1993.

WILLIS, P. Aprendiendo a trabajar. Madrid: Akal, 1988.

WHITTY, G. et al. La escuela, el estado y el mercado. Madrid, Morata, 1999. 